\title{
Terramicina en la Cavidad Abdominal
}

\author{
Dr. Alberto Zabaleta Lombana - Dr. Jorge Milanés*
}

Desde el pasado mes de Febrero hasta Junio del presente año, hemos verificado cincuenta observaciones en la Clínica Universitaria "Rafael Calvo C." sobre el uso del Clorhidrato de Oxitetraciclina, $(\mathrm{xx})$ en el acto operatorio de pacientes internadas en dicho servicio.

En general, con la llegada de los antibióticos se aprovechó su poder anti-infeccioso en el tratamiento de la peritonitis mediante su instilación en la cavidad abdominal. En 1955, entre nosotros, Alfonso Bonilla Naar (1) al cerrar el abdomen en las peritonitis generalizadas, dejaba una solución de Penicilina un millón de unidades y un gramo de Estreptomicina en 40 c.c. de agua destilada; además, drenes y otro antibiótico de amplio espectro por vía parenteral.

En 1956 William E. Schattenn (2) publicó sus observaciones sobre 38 casos de peritonitis generalizada mediante el uso de antibióticos intraperitoneal; de los cuales 20 lo fueron con Terramicina.

Para el efecto, una vez intervenido el paciente dejaba dos drenes, uno en la región subhepática derecha y otro a través del Douglas, mediante los cuales instilaba la solución antibiótica. Igual sistema de administración utilizaron A. Bonilla Naar, Alvarez Vásquez (1) en trabajo publicado en 196乞.

Experimentalmente se ha demostrado por observaciones sobre animales (2) (1) que los antibióticos en solución dejados en la cavidad abdominal no son tóxicos ni favorecen la aparición de adherencias.

Su administración en perros no anestesiados provoca la sensación de dolor cuando la concentración del producto es superior a los $2 \mathrm{mgm}$. por c.c. de solución. (2)

* Del "Departamento de Obstetricia" Universidad de Cartagena. 
Tal inconveniente no se presenta en los pacientes anestesiados o agregando a la solución antibiótica $2 \mathrm{mg}$. de ácido ascórbico por centímetro cúbico. (4)

METODO. - Preparamos una solución de Terramicina, tipo endovenoso; $250 \mathrm{mgm}$. en 15 c.c. de Dextrosa al $5 \%$ en agua destilada; para dejar en la cavidad peritoneal una vez verificada la intervención propuesta. En los casos considerados de pronóstico más grave utilizamos $500 \mathrm{mgm}$. de Terramicina en 20 c.c. de solvente. Al finalizar este trabajo nos servimos del suero fisiológico como solvente en cantidad de 30 centímetros.

Por lo demás, la Terramicina así preparada es la única medicación antibiótica que se administra; consignando expresamente en las órdenes médicas no aplicar ninguna otra droga de tal naturaleza en los días siguientes. Se continúan sí las medidas apropiadas de acuerdo con el estado y requerimiento del paciente: transfusiones, soluciones electrolíticas, calmantes de dolor etc. De igual manera, se toman todos los cuidados que son de rutina en el preoperatorio en la medida que la urgencia lo permita.

Este uso de la Terramicina en la cavidad abdominal prácticamente se ha hecho de rutina en la clientela quirúrgica del Servicio tanto civil como pensionada; habiéndose acortado con tal proceder su hospitalización. El estado general del paciente es mucho más satisfactorio: facies despejada, descenso marcado de la temperatura en las que han sido operadas en período febril, menor dolor a nivel de la herida; morbilidad postoperatoria reducida al mínimo, todo lo cual permite levantarlas con la confianza del enfermo, en las primeras 24 horas; y con las ventajas innegables de la movilización precoz.

En los casos observados no se ha presentado intolerancia de tipo alérgico ni síntoma abdominal alguno que interfiriera el postoperatorio.

Las intervenciones se verificaron mediante la raquianestesia; excepto en dos pacientes en las que debido a su mal estado general se recurrió a la anestesia local con Xilocaína.

La primera no toleró la solución; reaccionando con síntomas de lipotimia, sudoración, taquicardia; por lo que no llegó a inyectarse toda la cantidad (Historia: C. B. H.) ; y en la segunda, a pesar del trastorno acusado, se dejó toda la solución, terminán- 
dose de cerrar los planos. Esta sintomatología, no obstante lo inconveniente e inesperada, no tuvo mayores consecuencias, pasando en algunos minutos.

El tipo de anestesia usado, el solvente: agua destilada y la alta concentración de Terramicina pudieran provocar este malestar al entrar en contacto con los tejidos lesionados. Por ello, en las últimas pacientes cambiamos al suero fisiológico en cantidad de 30 centímetros.

De esta manera, completamos 50 tratamientos cuyo estudio resumimos en las historias y cuadros que acompañan este trabajo.

Para efecto de las anotaciones, consideramos la hora en que termina la intervención como comienzo del postoperatorio; y las 8 de la mañana del día de salida (hora en que generalmente se pasa la visita reglamentaria) como la de su término hospitalario.

Solamente dos de estos 50 casos, ambos operados en los mismos días y vecinos de cama, presentaron complicación bronconeumónica, lo que obligó al uso de antibióticos parenteral, dominándose rápidamente el cuadro. (Historias Nos. 02305 y 02106 que figuran con más detalles en los apartados siguientes).

Hemos incluído la Historia N⿳0 01684, paciente que no fue operada; y atendida con el diagnóstico de pelviperitonitis aguda. Previa punción del Douglas con extracción de aproximadamente 1 c.c. de líquido seropurulento, se inyectó por la misma vía los 15 c.c. de solución de Terramicina en Dextrosa al 5\% en agua destilada con resultados realmente notables.

Esta paciente acusaba dolor intolerable en el bajo vientre que dificultaba la exploración, tem. de 39.5; y además blefaritis bilateral. Antes de las primeras 24 horas había desaparecido toda sintomatología; incluso la blefaritis, dándosele de alta al siguiente día completamente bien.

No hemos hecho selección alguna de los casos para tratamiento, siendo tan solo desechados aquellos que por cualquier causa recibieran antibióticos antes de ser intervenidos. Las operaciones fueron llevadas a cabo por los profesores y jefes de Clínica, cinco en total; muchas de ellas con el carácter de urgente, vale decir, sin tiempo para una buena preparación preoperatoria.

Historiamos estas 50 observaciones en tres cuadros: Cesáreas Segmentarias Transperitoneales, Embarazos Ectópicos Rotos y Hematosalpinx. Con el título de "Varios" encuadramos los 
CUADRO No 1

\section{Cesáreas segmentarias transperitoneales}

\begin{tabular}{|c|c|c|c|c|c|c|c|}
\hline \multirow{2}{*}{ HIST. No } & \multirow{2}{*}{ CAUSA } & \multirow{2}{*}{$\begin{array}{l}\text { T. de P. } \\
\text { HORAS }\end{array}$} & \multicolumn{4}{|c|}{ POST. OPERAT. Mx. TEMPERAT. } & \multirow{2}{*}{ OBSERVACIONES } \\
\hline & & & DIAS & HORAS & GRADO & HORAS & \\
\hline 02479 & Cesárea Anterior & 0 & 1 & 20 & 37.6 & 28 & $\begin{array}{l}\text { G.2. - P.O. } 33 \text { años. Cesárea anterior por preclampsia hace } 18 \\
\text { meses. Está en tratamiento con hipotensores. }\end{array}$ \\
\hline 02337 & Sit. Transversa & 28 & 3 & 2 & 38 & 24 & G3. - P.2. - A las 48 horas de operada solicitaba su salida. \\
\hline 02106 & $\begin{array}{l}\text { Oclusión Vaginal } \\
1 / 3 \text { superior }\end{array}$ & $\begin{array}{l}\text { más } \\
10\end{array}$ & 5 & 20 & 38.8 & 52 & $\begin{array}{l}\text { Hrimigrávida, } 18 \text { años. A las } 48 \text { horas acusa dolor torácico, esca- } \\
\text { lofríos, fiebre, estertores cripitantes ambos pulmones. Bronconeu- } \\
\text { monía. Leucccitos } 18.550 \text {. Hematíes: } 2.480 .000 \text {. Hb: } 50 \% \text {. Se } \\
\text { utilizó antibiótico parenteral. }\end{array}$ \\
\hline 02305 & PI. Previa Cent. & 0 & 5 & 4 & 39.8 & 36 & $\begin{array}{l}\text { G.9. P 5-A } 331 \text { años. Malas condiciones nutricional y dentaria. } \\
\text { Pesó } 46 \mathrm{kl} \text {. Talla } 1.60 \text {. Entró a cirugía con } 38.3 \text { temp. Hizo bron- } \\
\text { coneumonía. Vecina de cama de la anterior. Se usó antibiótico } \\
\text { en el postoperatorio. Hematíes: } 2.910 .000 \text {. Hb. } 55 \% \text {. }\end{array}$ \\
\hline 02186 & $\begin{array}{l}\text { Procidencia } \\
\text { de cordón }\end{array}$ & $\begin{array}{l}\text { más } \\
10\end{array}$ & 4 & 13 & 38.4 & 45 & $\begin{array}{l}\text { G.12 P.9 A.2 } 38 \text { años. Hidrohematorrea } 3 \text { días antes de ingreso. } \\
2.580 .000 \text { hematíes. } 50 \% \text { de hemoglobina. Leucocitos: } 18.200 \text {. }\end{array}$ \\
\hline 01914 & $\begin{array}{l}\text { T. de P. } \\
\text { prolongado }\end{array}$ & 50 & 4 & 16 & 37.8 & 24 & $\begin{array}{l}\text { F'rimigrávida } 16 \text { años. Llegó con bolsa rota, considerable edema } \\
\text { de la vulva, meconio fétido. Atendida por comadrona. Temperatu- } \\
\text { ra } 37.5^{\circ} \text {. }\end{array}$ \\
\hline 09197 & Proc. de cordón & 9 & 3 & 2 & 37.5 & 36 & G.9 P.7 A 1 - 32 años. Hizo la procidencia en el servicio. \\
\hline 02184 & $\begin{array}{l}\text { T. de P. prolonga- } \\
\text { do. Suf. fetal }\end{array}$ & 27 & 3 & 7 & 37.6 & 60 & $\begin{array}{l}\text { G.2 P.1 } 18 \text { años. Embarazo gemelar no diagnosticado. Hematíes: } \\
3.140 .000 \text {. Hemog.: } 60 \% \text {. Leucocitos: } 20.600 \text {. }\end{array}$ \\
\hline 02844 & PI. Previa Cent. & 0 & 3 & 17 & 37.7 & 13 & G.12 P.5 A.6 34 años. Hematíes: 3.990.000. Hemoglb.: $75 \%$. \\
\hline 03217 & Primg. Añosa & 9 & 1 & 12 & 37.3 & 16 & Primigrávida. 35 años. Paciente pensionada. \\
\hline A.T.Ch & $\begin{array}{l}\text { Cesáreas Anteriores } \\
\text { por estenosis pélvica }\end{array}$ & - & 2 & 0 & 37.5 & 30 & $\begin{array}{l}\text { G.3 P.0 - } 25 \text { años. Dos embarazos anteriores terminados por cesá- } \\
\text { reas. Feto sexo femenino. } 3.450 \mathrm{grm} \text {. Talla } 49 \mathrm{~cm} \text {. Terramicina } \\
\text { en solución tisiológica: } 15 \text { c.c. }\end{array}$ \\
\hline
\end{tabular}


CUADRO N: 1

\section{Cesáreas segmentarias transperitoneales}

\begin{tabular}{|c|c|c|c|c|c|c|c|}
\hline \multirow{2}{*}{ HIST. No } & \multirow{2}{*}{ CAUSA } & \multirow{2}{*}{$\begin{array}{l}\text { T. de P. } \\
\text { HORAS }\end{array}$} & \multicolumn{4}{|c|}{ POST. OPERAT. MX. TEMPERAT. } & \multirow[b]{2}{*}{ OBSERVACIONES } \\
\hline & & & DIAS & HORAS & GRADO & HORAS & \\
\hline 02629 & $\begin{array}{l}\text { Cesárea corporal } \\
\text { ant. Preruptura } \\
\text { uterina }\end{array}$ & 6 & 2 & 0 & 38.5 & 30 & $\begin{array}{l}\text { G.2 P.0 - } 24 \text { años. Primer embarazo: cesárea corporal y peritoni- } \\
\text { tis. } 11 \text { meses más tarde, segundo embarazo. Hay dehiscencia de } \\
\text { rectos y hernia. Kahn positivo. Hematocrito } 34 \% \text {. Hemog.: } 60 \% \\
\text { Adherencias epiploicas. Hematoma a nivel de la cicatriz uterina a } \\
\text { punto de romperse. Se elimina la antigua cicatriz después de cesá- } \\
\text { rea segmentaria. Feto eritroblastósico. }\end{array}$ \\
\hline E. C. & PI. Previa Cent. & 3 & 2 & 0 & 37.5 & 30 & $\begin{array}{l}\text { G.2 P.1 - } 22 \text { años. Tres días antes, sangre abundante. En el ser- } \\
\text { vicio: palidez, angustia marcadas. Algunos vómitos. T.A.: } 80 \text { x } \\
50 \text {, pulso 120. Se transfunde } 500 \text { c.c. de sangre. Se interviene. } \\
\text { Al segundo día: } 2.490 .000 \text { hematíes. } 22 \% \text { hematocrito. } 40 \% \text { he- } \\
\text { mogl. Terramicina en sol. fisiológica. }\end{array}$ \\
\hline 02248 & $\begin{array}{l}\text { Pl. Previa. } \\
\text { Sit. Transversa }\end{array}$ & 0 & 1 & 20 & 37.2 & 28 & $\begin{array}{l}\text { Edad } 39 \text { años. Llegó al servicio por hemorragia vaginal indolora. } \\
\text { Embarazo a término. Situación fetal transversa. Bolsa intacta. }\end{array}$ \\
\hline I.C.C. & Cesárea Anterior & 4 & 1 & 21 & 37.8 & 9 & $\begin{array}{l}\text { G.8 P.6 Ultimo embarazo: cesárea por placenta previa. Esta vez } \\
\text { se interviene por la cesárea anterior. Edad } 34 \text { años. Buen estado } \\
\text { general. Bolsa intacta. Feto femn. } 4.100 \mathrm{gr} \text {. Talla } 50 \mathrm{cms} \text {. }\end{array}$ \\
\hline P.E.R. & PI. Previa & 0 & 1 & 20 & 37.5 & 28 & $\begin{array}{l}\text { G.8 P.7 - } 32 \text { años. Bolsa intacta. Buen estado general. } 32 \text { sema- } \\
\text { nas de embarazo aproximadamente. }\end{array}$ \\
\hline M.C.M. & $\begin{array}{l}\text { Sit. Fetal Transversa } \\
\text { Suf. Fetal }\end{array}$ & 32 & 2 & 10 & 38 & - & $\begin{array}{l}\text { G.11 P.10. L'egó al servicio después de } 29 \text { horas de t. de parto. } \\
\text { Bolsa intacta. Se rompe. El interno reconoce la posición anóma- } \\
\text { la. Tres horas más tarde cirugía. Feto: } 3.300 \text { gr. Pérdida regu- } \\
\text { lar de sangre. Se transfundieron } 500 \text { c.c. Entró a cirugía con } \\
38 \text { grados de temperatura. que cayó a partir de las } 40 \text { horas. } \\
\text { Estado catarral con expectoración. Se dejó terramicina: } 250 \text { mmg. } \\
\text { en } 30 \text { c.c. de sol. fisiológica. }\end{array}$ \\
\hline
\end{tabular}


CUADRO N: 1

\section{Cesáreas segmeniarias transperitoneales}

\begin{tabular}{|c|c|c|c|c|c|c|c|}
\hline \multirow{2}{*}{ HIST. N No } & \multirow[b]{2}{*}{ CAUSA } & \multirow{2}{*}{$\begin{array}{l}\text { T. de P. } \\
\text { HORAS }\end{array}$} & \multicolumn{4}{|c|}{ POST. OPERAT. Mx. TEMPERAT. } & \multirow[b]{2}{*}{ OBSERVACIONES } \\
\hline & & & DIAS & HORAS & GRADO & HORAS & \\
\hline 3.497 & $\begin{array}{l}\text { T. de P. prolong. } \\
\text { Present. de cara }\end{array}$ & 31 & 3 & 10 & - & - & $\begin{array}{l}\text { G.3 P.2 - } 34 \text { años. Bolsa rota. Hipersistolia. Entra a cirugía con } \\
38^{\circ} \text { de temperatura. Descendió a las } 48 \text { horas. }\end{array}$ \\
\hline 3.585 & $\begin{array}{l}\text { Desprendimiento pre- } \\
\text { maturo placenta } \\
\text { normoinserta }\end{array}$ & - & 5 & 18 & 39.4 & 48 & $\begin{array}{l}\text { G.5 P.3 A.1 } 27 \text { años. Prueba de t. de parto durante } 4 \text { horas in- } \\
\text { ducido. Se intervino por hemorragia. } 28 \text { semanas aproximadamen- } \\
\text { te de embarazo. Estado catarral. Postoperatorio: estertores crepi- } \\
\text { tantes pulmonares. Se usó Penicilina parenteral. Hematíes: } \\
3.990 .000 \text {. Hemog.: } 35 \% \text {. Hematocr.: } 18 \% \text {. Leucocitos: } 13.800 \text {. }\end{array}$ \\
\hline 3.644 & $\begin{array}{l}\text { Cesárea anterior por } \\
\text { PI. previa }\end{array}$ & 3 & 3 & 18.5 & 37.8 & 60 & $\begin{array}{l}\text { G.3 P.0 A.1 Primer embarazo: placenta previa y cesárea. A los } \\
4 \text { meses: nuevo embarazo y aborto. Estado catarral. Eventración } \\
\text { a nivel de la cicatriz laparotómica. Numerosas adherencias epi- } \\
\text { ploicas. Raquianestesia que termina con aplicación de pentotal. } \\
\text { Espasmo larírigeo y mal estado general de la paciente. }\end{array}$ \\
\hline J.N.A. & $\begin{array}{l}\text { T. de P. } \\
\text { prolongado } \\
\text { Preeclampsia } \\
\text { severa }\end{array}$ & 30 & 3 & 0 & 37.8 & 60 & $\begin{array}{l}\text { Primigrávida } 23 \text { años. T.A. } 195 \times 130 \text {. Pulso } 120 \text {. Temp.: } 38.4^{\circ} \text {. } \\
\text { Bolsa rota } 4 \text { horas antes. Anotados } 10 \text { tactos vaginales. Se utili- } \\
\text { zó hipotensores. Anestesia local con Xilocána. La solución de Te- } \\
\text { rramicina prcvocó lipotimia que duró algunos minutos. Al salir } \\
\text { de cirugía: T.A.: } 90 \times 50 \text {. Pulso } 132 \text {. A las } 12 \text { horas tempera- } \\
\text { tura } 36.6^{\circ} \text {. }\end{array}$ \\
\hline $\begin{array}{l}\text { E. P. } \\
\text { de T. }\end{array}$ & T. de P. prolongado & 31 & 1 & 21 & 37.4 & 24 & $\begin{array}{l}\text { Primigrávida pensionada. } 29 \text { años. Más de } 24 \text { horas con bolsa ro- } \\
\text { ta. Se anotan } 5 \text { exámenes vaginales para control de trabajo. Se } \\
\text { usó la Terramicina en solución fisiológica } 15 \text { c.c. }\end{array}$ \\
\hline L.C.C. & $\begin{array}{l}\text { T. de P. prolongado } \\
\text { Pres.: de Cara }\end{array}$ & 32 & 8 & 21 & 38 & 24 & $\begin{array}{l}\text { G.6 P.5 - Entró a cirugía con temperatura } 38.3^{\circ} \text { Extracción fetal } \\
\text { bastante laboriosa. A las } 48 \text { horas nueva elevación térmica que } \\
\text { coincide con congestión mamaria pcr bajada de la leche. Terra- } \\
\text { micina en suero fisiológico } 15 \text { c.c. }\end{array}$ \\
\hline
\end{tabular}


CUADRO N: 2

\section{Embarazos ectópicos rotos. - Hematosalpinx}

\begin{tabular}{|c|c|c|c|c|c|c|}
\hline \multirow[b]{2}{*}{ HIST. Ne } & \multirow[b]{2}{*}{ INTERVENCIONES } & \multicolumn{4}{|c|}{ POST. OPERAT. MX. TEMPERAT. } & \multirow[b]{2}{*}{ OBSERVACIONES } \\
\hline & & DIAS & HORAS & GRADO & HORAS & \\
\hline 01675 & $\begin{array}{l}\text { Salpingooforect. } \\
\text { Der. Apendicect. } \\
\text { profiláctica }\end{array}$ & 2 & 21 & 37.3 & 21 & $\begin{array}{l}\text { G.6 P.4 - Hace dos años operada por ectópico izq. } 32 \text { años. Metro- } \\
\text { rragia desde hace } 1 \text { mes, dolor hipogástrico, peso rectal. Punción } \\
\text { del Douglas positiva. }\end{array}$ \\
\hline 01961 & Salpingooforect. Izq. & 3 & 16 & 38.5 & 40 & $\begin{array}{l}\text { G.4 P.3 - } 25 \text { años. Metrorragia desde hace } 3 \text { días. Fondo de saco } \\
\text { de Douglas doloroso. Hematosalpinx Izquierdo. }\end{array}$ \\
\hline 01691 & Salpingooforect. Der. & 3 & 20 & 38.1 & 28 & $\begin{array}{l}\text { G.11 P.10 - } 39 \text { años. En cavidad abdominal líquido serosanguíneo } \\
\text { y coágulos. Adherencias. Feto de } 7 \mathrm{cms} \text {. Raquianestesia y al final } \\
\text { pentotal para el cierre de los planos. }\end{array}$ \\
\hline 01818 & $\begin{array}{l}\text { Salpingect. Derecha } \\
\text { Apendicect. profil. }\end{array}$ & 4 & 17 & - & - & $\begin{array}{l}\text { G.4 P.2 A.1 - } 28 \text { años. Metrorragia de regular intensidad durante } \\
1 \text { semana. Curetaje por considerarse aborto incompleto, restos } \\
\text { mal olientes. Entró a cirugía con temperatura } 38.9^{\circ} \text { Gran canti- } \\
\text { dad de coágulos negros. Encerrado en sus membranas feto de } 20 \\
\mathrm{cms} \text {. Placenta incertada en porción istmica trompa derecha, en } \\
\text { parte desgarrada. Hematíes: } 2.700 .000 \text {. Hemog.: } 40 \% \text {. Embara- } \\
\text { zo Istmico abdominal. }\end{array}$ \\
\hline 02316 & Salpingect. Derecha & 2 & 18 & 37.6 & 48 & $\begin{array}{l}\text { G.4 P.3 - } 24 \text { años. Sintomatología } 4 \text { días antes. Punción de Dou- } \\
\text { glas positiva. Coágulos y sangre líquida en abdomen. Se visualiza } \\
\text { huevo formando tumoración en trompa derecha. Se hace necesa- } \\
\text { rio completar anestesia con pentotal. }\end{array}$ \\
\hline 03025 & Salpingect. Derecha & 3 & 7 & 37.8 & 60 & $\begin{array}{l}\text { G.6 P.4 A.1 - } 33 \text { años. Sintomatología desde } 13 \text { días. Curetaje } \\
\text { por considerarse aborto incompleto. Punción de Douglas positiva. } \\
\text { Sangre líquida y coágulos en abdomen. Se hizo raquianestesia, } \\
\text { pentotal y éter. }\end{array}$ \\
\hline 3.643 & $\begin{array}{l}\text { Salpingect. Derecha } \\
\text { Apendicect. profil. }\end{array}$ & 8 & 3 & 37.5 & 36 & $\begin{array}{l}\text { G.4 P.3 - } 27 \text { años. Pesó } 41.3 \text { klg. Talla: } 1.46 \mathrm{cms} \text {. Metrorragia } \\
\text { desde } 16 \text { días antes. Punción de Douglas positiva. Abundante san- } \\
\text { gre líquida y coágulos en abdomen. Hematíes: } 2.680 .000 \text {. He- } \\
\text { mog.: } 45 \% \text {. }\end{array}$ \\
\hline
\end{tabular}


CUADRO N $N^{\circ} 2$

Embarazos ectópicos rotos. - Hematosalpinx

\begin{tabular}{|c|c|c|c|c|c|c|}
\hline \multirow{2}{*}{ HIST. N No } & \multirow{2}{*}{ INTERVENCIONES } & \multicolumn{4}{|c|}{ POST. OPERAT. Mx. TEMPERAT. } & \multirow[b]{2}{*}{ OBSERVACIONES } \\
\hline & & DIAS & HORAS & GRADO & HORAS & \\
\hline 3.391 & $\begin{array}{l}\text { Salpingect. Derecha } \\
\text { Apendicect. profil. }\end{array}$ & 2 & 17 & - & - & $\begin{array}{l}\text { Primigrávida. } 22 \text { años. Pesó } 40 \mathrm{klg} \text {. Talla: } 1.56 \mathrm{cms} \text {. Una sema- } \\
\text { na antes hospitalizada en malas condiciones generales por provoca- } \\
\text { ción de aborto. Se hizo transfusión y dio de alta mejorada. Regre- } \\
\text { só en peores condiciones, siendo intervenida. Abundante sangre } \\
\text { en abdomen Entró a cirugía con temperatura } 38.5^{\circ} \text { desaparecien- } \\
\text { do antes de las } 24 \text { horas. Hematocrito: } 19 \% \text {. }\end{array}$ \\
\hline A.M.Z. & $\begin{array}{l}\text { Slpingect. bilateral } \\
\text { Enucleación quiste } \\
\text { ovario derecho }\end{array}$ & 2 & 21 & 38 & 28 & $\begin{array}{l}\text { G.7 P.6 - } 35 \text { años. Pequeñas metrorragias desde hace } 1 \text { mes. } \\
\text { Buen estado general. Hematosalpinx izquierdo. Salpingitis quística } \\
\text { derecha. Quiste de ovario derecho. }\end{array}$ \\
\hline $\begin{array}{l}\text { A. J. } \\
\text { de S. }\end{array}$ & Salpingect. Izq. & 1 & 19 & 38.5 & 4 & $\begin{array}{l}33 \text { años. Sintomatología dolorosa desde una semana antes. Pun- } \\
\text { ción de Douglas positiva. Entra a cirugía con } 38^{\circ} \text { temp. Abundan- } \\
\text { te sangre en cavidad abdominal. Se liberan adherencias. Hemato- } \\
\text { salpinx izquierdo. La temperatura descendió a } 37.5^{\circ} \text { a las } 19 \text { ho- } \\
\text { ras. Terramicina: } 250 \mathrm{mmg} \text {. en sol. fisiológica } 30 \text { c.c. }\end{array}$ \\
\hline L.P.C. & Salpingect. Izq. & 1 & 16 & 37.8 & 24 & $\begin{array}{l}\text { G.4 P. } 3 \text { - Sir tomatología metrorrágica y dolorosa desde hace } 3 \\
\text { días. Edad: } 25 \text { años. Punción de Douglas positiva. Coágulos y san- } \\
\text { gre líquida en cavidad abdominal. Hematosalpinx izquierdo. He- } \\
\text { mog.: } 7 \text { gr. Hematocrito: } 20 \% \text {. Terramicina: } 250 \text { mmg. en } 30 \\
\text { c.c. sol. fisióógica. }\end{array}$ \\
\hline
\end{tabular}


CUADRO N: 3

Varios

\begin{tabular}{|c|c|c|c|c|c|c|c|}
\hline \multirow{2}{*}{ HIST. № } & \multirow{2}{*}{ CAUSA } & \multirow{2}{*}{ INTERVENCIONES } & \multicolumn{2}{|c|}{ POST. OPERAT. } & \multicolumn{2}{|c|}{ Mx. TEMPERAT. } & \multirow{2}{*}{ OBSERVACIONES } \\
\hline & & & DIAS & HORAS & GRADO & HORAS & \\
\hline 02159 & Perforación uterina & Histerorafia & 2 & 7 & 37.7 & 16 & $\begin{array}{l}\text { Al practicar curetaje por aborto incompleto se provoca la perfo- } \\
\text { ración. } 1.5 \mathrm{cms} \text {. en fondo. Se sutura con catgut. }\end{array}$ \\
\hline 01622 & Perforación uterina & $\begin{array}{l}\text { Histerorafia } \\
\text { Resección cuneiforme } \\
\text { armbos ovarios }\end{array}$ & 2 & 18 & 37.8 & 28 & $\begin{array}{l}\text { Paciente pensionada. Sangre considerable y coágulos en abdomen. } \\
\text { Hacia cuerno izq. perforación } 1 \mathrm{~cm} \text {. Entre ligamentos útero sa- } \\
\text { cros otra perforación de } 2 \mathrm{cms} \text {. anfractuosa. }\end{array}$ \\
\hline 01458 & $\begin{array}{l}\text { Quiste ovario } \\
\text { derecho }\end{array}$ & Ooforectomía & 1 & 22 & 37.5 & 30 & \\
\hline 02422 & $\begin{array}{l}\text { Quiste ovario lzq. } \\
\text { con pedículo torcido }\end{array}$ & Ooforectomía & 4 & 9 & - & - & $\begin{array}{l}\text { Sintomatología dos días antes. Llega a cirugía con } 39.8^{\circ} \text { temp. Al } \\
\text { abrir peritonso cae en shock impidiendo continuar durante } 15 \mathrm{mi}- \\
\text { rutos. Se transfundió sangre } 500 \text { c.c. Quiste azuloso con pedículo } \\
\text { algo necrótico. }\end{array}$ \\
\hline 00405 & $\begin{array}{l}\text { Absceso Anexo } \\
\text { Derecho }\end{array}$ & $\begin{array}{l}\text { Salpingooforectomía } \\
\text { Apendicectomía }\end{array}$ & 2 & 21 & 38.2 & 48 & $\begin{array}{l}\text { Curetaje por aborto incompleto. Regresa a la semana con sínto- } \\
\text { mas de absceso pélvico. Colpotomía posterior que no llena su co- } \\
\text { metido. Posteriormente cirugía: fuertes adherencias. Se drena el } \\
\text { absceso. Terramicina: } 500 \mathrm{mmg} \text {. Apendicectomía. }\end{array}$ \\
\hline 01684 & $\begin{array}{l}\text { Pelviperitonitis } \\
\text { Aguda }\end{array}$ & $\begin{array}{l}\text { Inyección a través } \\
\text { de!! Douglas }\end{array}$ & 1 & 21 & - & - & $\begin{array}{l}\text { Temperatura } 39.5^{\circ} \text { Dolor difuso en hipogastrio con resistencia mus- } \\
\text { cular. Punción del Douglas: líquido seropurulento. Por la misma } \\
\text { váa se dejan } 250 \mathrm{mmg} \text {. Terramicina a las } 2 \mathrm{p} \text {. } \mathrm{m} \text {. A las } 4 \mathrm{a} \text {. m. } \\
\text { del siguiente día, temperatura } 36.5^{\circ} \text { Desapareció dolor e igual- } \\
\text { mente una blefaritis bilateral. }\end{array}$ \\
\hline 02426 & $\begin{array}{l}\text { Piosalpinx } \\
\text { Bilateral }\end{array}$ & $\begin{array}{l}\text { Salpingect. Der. } \\
\text { Salpingooforect. } \\
\text { Izq. Histerectomía } \\
\text { total }\end{array}$ & 5 & 20 & 39 & 48 & $\begin{array}{l}\text { Numerosas adherencias. Los abscesos derraman en la cavidad ab- } \\
\text { dominal al liberarlos. La intervención duró } 3 \text { hroas. Anestesia pe- } \\
\text { ridural, además, pentotal y éter. Al finalizar la paciente cayó en } \\
\text { shock, recuperándose al cabo de dos horas mediante transfusio- } \\
\text { nes. Se dejó } 500 \mathrm{mmg} \text {. Terramicina. }\end{array}$ \\
\hline
\end{tabular}


CUADRO No 3

Varios

\begin{tabular}{|c|c|c|c|c|c|c|c|}
\hline \multirow[b]{2}{*}{ HIST. N No } & \multirow[b]{2}{*}{ CAUSA } & \multirow[b]{2}{*}{ INTERVENCIONES } & \multirow{2}{*}{\multicolumn{2}{|c|}{\begin{tabular}{l|l}
\multicolumn{2}{|c|}{ POST. } \\
OPERAT. \\
DIAS & HORAS
\end{tabular}}} & \multicolumn{2}{|c|}{ Mx. TEMPERAT. } & \multirow[b]{2}{*}{ OBSERVACIONES } \\
\hline & & & & & GRADO & HORAS & \\
\hline 03238 & $\begin{array}{l}\text { Quiste ovario } \\
\text { Izquierdo }\end{array}$ & $\begin{array}{l}\text { Ooforectomía } \\
\text { Apendicect. prof. }\end{array}$ & 1 & 22 & - & - & \\
\hline 01423 & Tumor Previo & $\begin{array}{l}\text { Cesárea Segm. } \\
\text { Histerect. total }\end{array}$ & 2 & 20 & 37.4 & 52 & $\begin{array}{l}\text { G.7 P.5 - } 31 \text { años. Embarazo a término. T. de parto. A través } \\
\text { del cuello masa dura, sésil, del tamaño del puño. Histerectomía } \\
\text { total, previa cesárea segmentaria. Hematíes: } 1.860 .000 \text {. Hemato- } \\
\text { crito: } 16 \%\end{array}$ \\
\hline 02849 & $\begin{array}{l}\text { Quiste dermoide } \\
\text { Salpingitis } \\
\text { quística Izq. }\end{array}$ & $\begin{array}{l}\text { Salpingooforect. Izq. } \\
\text { Resección cuneiforme } \\
\text { ovario Der. } \\
\text { Apendicect. prof. }\end{array}$ & 3 & 22 & 38.3 & 6 & \\
\hline 01870 & Endometrioma? & $\begin{array}{l}\text { Ovariectom. Der. } \\
\text { Apendicectomía }\end{array}$ & 2 & 19 & 37.6 & 4 & $\begin{array}{l}\text { Hace } 6 \text { años salpingoplastia y resección cuneiforme ambos ova- } \\
\text { rios. Hay gren cantidad de adherencais. Se punciona el quiste de } \\
\text { contenido achocolatado y se extirpa la bolsa. Raquianestesia que } \\
\text { termina con pentotal. }\end{array}$ \\
\hline 3.191 & Absceso Pélvico & $\begin{array}{l}\text { Histerect. total } \\
\text { Salpingooforect. } \\
\text { Izq. Salpingect. Der. }\end{array}$ & 2 & 21 & 38.2 & 48 & $\begin{array}{l}\text { Talla: } 1.54 \text { Peso: } 48 \mathrm{klg} \text {. Vómitos el mismo día de operada con } \\
\text { expulsión de numerosos áscaris. Durante la nitervención el absce- }\end{array}$ \\
\hline 02869 & $\begin{array}{l}\text { Endometrioma? } \\
\text { Quiste luteínico }\end{array}$ & $\begin{array}{l}\text { Resección cuneif. } \\
\text { Ovar. Der. Apen- } \\
\text { dicect. profiláct. }\end{array}$ & 1 & 20 & 37.4 & 4 & \\
\hline G.Z.V. & Absceso pélvico & $\begin{array}{l}\text { Aspiración. Salpin- } \\
\text { gooforect. Der. }\end{array}$ & 4 & 16 & - & - & $\begin{array}{l}\text { Edad } 18 \text { años. } 40 \mathrm{klg} \text {. de peso. Aborto provocado mediante son- } \\
\text { da. En cavidad abdominal derrame de pus mal oliente; aspirán- } \\
\text { dose casi 1. Apelotonamiento de asas intestinales que conservan } \\
\text { su disposición anatómica. Temperatura } 39.5^{\circ} \text { que cayó definiti- Tou } \\
\text { vamente a las } 60 \text { horas. Por la gravedad en principio se le apli- } \\
\text { có en venoclisis } 250 \mathrm{mmg} \text {. más de terramicina. }\end{array}$ \\
\hline
\end{tabular}




\section{CUADRO No 3}

\section{Varios}

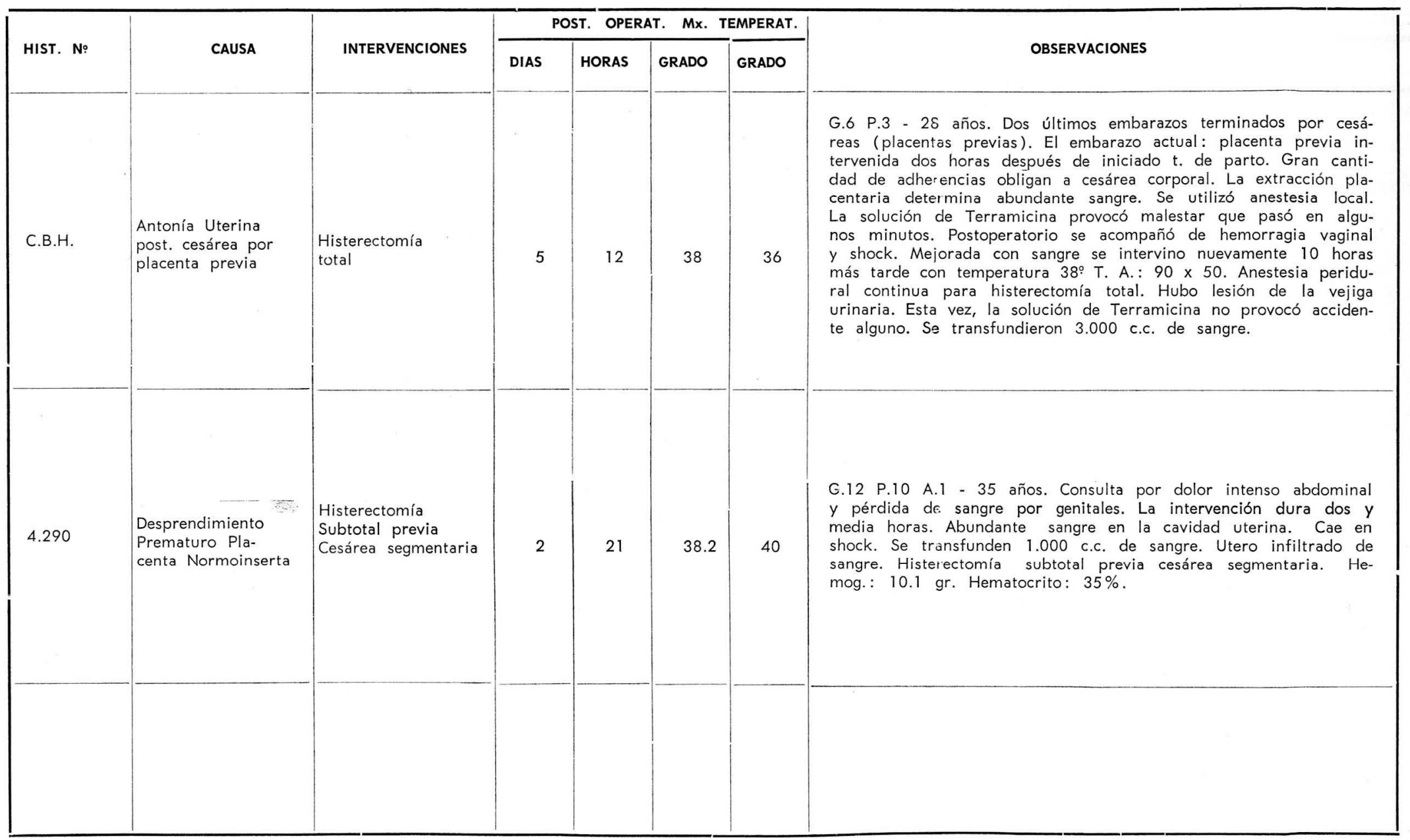


CUADRO Nং 4

Totales parciales

\begin{tabular}{|c|c|c|c|c|c|c|c|c|}
\hline \multirow{2}{*}{ INTERVENCIONES } & \multirow{2}{*}{$\begin{array}{l}\text { No DE } \\
\text { CASOS }\end{array}$} & \multirow{2}{*}{$\begin{array}{l}\text { PORCEN- } \\
\text { TAJES }\end{array}$} & \multicolumn{2}{|c|}{ POSTOPERATORIO } & \multicolumn{3}{|c|}{ TIEMPO PROMEDIO } & \multirow{2}{*}{$\begin{array}{c}\text { HORAS } \\
\text { PROMEDIO }\end{array}$} \\
\hline & & & DIAS & HORAS & DIAS & HORAS & MINUT. & \\
\hline Cesáreas Segm. & 23 & $46 \%$ & 72 & 8 & 3 & 3 & 30 & $751 / 2$ \\
\hline Ectópicos etc. & 11 & $22 \%$ & 33 & 12 & 3 & 1 & 05 & $73.05^{\prime}$ \\
\hline Varios & 16 & $32 \%$ & 51 & 3 & 3 & 4 & 41 & $76.41^{\prime}$ \\
\hline \multicolumn{9}{|c|}{ CUADRO N 5} \\
\hline Gran Total & 50 & $100 \%$ & 156 & 23 & 3 & 3 & 20 & $75.1 / 4$ \\
\hline
\end{tabular}


casos de abscesus pélvicos, quistes de ovario, perforaciones uterinas por curetajes, histerectomías etc. En las respectivas columnas anotamos los días y horas del postoperatorio de acuerdo con lo indicado anteriormente a este respecto, causa y tipo de intervención, como también el más alto grado de temperatura alcanzado y el número de horas transcurrido al producirse esta hipertermia. La última columna recoge los antecedentes y un resumen de las condiciones pre y postoperatorias. Finalmente, los cuadros 4 y 5 agrupan en forma parcial y total los datos generales de este estudio.

No queremos alargarnos en comentarios sobre las historias y cuadros que presentamos, ya que nada agregaríamos a su objetividad. Queremos sí recalcar el beneficio obtenido con este tratamiento que permitió una permanencia hospitalaria promedio de solo 3 días, 3 horas y 20 minutos por paciente en 50 casos, muchos de los cuales de notable gravedad; y la mayoría en pésimas condiciones preoperatorias.

\section{RESUMEN}

1) Se presentan 50 casos de diferentes intervenciones laparotómicas obstétrico-ginecológicas, en las cuales se dejó 250-500 mgm. de terramicina tipo endovenoso en 15-20 y 30 centímetros de dextrosa al $5 \%$ en agua destilada y en solución fisiológica, en la cavidad abdominal.

2) Se revisa la literatura sobre la Terramicina intraperitoneal. Observamos que la misma se refiere al tratamiento de la peritonitis generalizada mediante perfusión abdominal.

3) Se presenta un caso en el cual se usó la Terramicina en 15 c.c. de solución inyectada a través del fondo de saco de Douglas.

4) Se acompañan dos cuadros estadísticos que revelan la bondad del procedimiento.

\section{CONCLUSIONES}

1) La Terramicina en solución dejada en la cavidad abdominal es un procedimiento completamente inocuo y altamente eficaz en la prevención de la morbilidad post-operatoria. 
2) Permite acortar notablemente el internamiento postoperatorio hospitalario.

3) Permite por lo tanto, mayor aprovechamiento del cupo hospitalario a un costo menor por paciente y un gasto de antibióticos reducido al mínimo.

4) El estado general del paciente dentro de las primeras 24 horas es más satisfactorio, aceptando con confianza la movilización precoz.

5) Solo se presentaron dos complicaciones por bronconeumonía, lo que obligó al uso de antibióticos por vía parenteral.

6) Este tratamiento no debe utilizarse en los casos intervenidos con anestesia local por dar origen a percances desagradables.

Nuestros agradecimientos al personal Científico de Clínica de Maternidad Doctores: José María Pérez Ruiz, Orlando Castillo C., Boris Calvo del Río y Roberto Dickson.

\section{BIBLIOGRAFIA}

1) BONILLA NAAR A., ALVAREZ VASQUEZ A. Tratamiento Racional de la Peritonitis Generalizada. Rev. Med. y C.irugía Vol. XXIV. № 5. Oct.-Nov. 1960.

2) SCHATTENN W. E. Intraperitoneal Antibiotic Administration. Surg. Gynec. and Obst. 102 Marzo/56.

3) DI CARLO G. Terramycin Therspy. Pág. 109. Editor Medical Department of Pfizer Laboratories.

4) EDER W. P. and MYERS N. L. Terramycin Therapy. Pág. 110. Editor Medical Department of Pfizer Laboratories. 1960. U.S.A.

5) SCHATTENN W. E. and ABBOTT W. E. Terramycin Therapy. Pág. 109. Editor: Medical Department of Pfizer Lab. 1960. U. S. A. 